\title{
Persistent currents in diffusive metallic cavities: Large values and anomalous scaling with disorder
}

\author{
G. Chiappe and M. J. Sánchez \\ Departamento de Física J. J. Giambiagi, \\ Facultad de Ciencias Exactas y Naturales, \\ Universidad de Buenos Aires. Ciudad Universitaria, 1428 Buenos Aires, Argentina.
}

\begin{abstract}
The effect of disorder on confined metallic cavities with an Aharonov-Bohm flux line is addressed. We find that, even deep in the diffusive regime, large values of persistent currents may arise for a wide variety of geometries. We present numerical results supporting an anomalous scaling law of the average typical current $<I_{t y p}>$ with the strength of disorder $w,<I_{t y p}>\sim w^{-\gamma}$ with $\gamma<2$. This is contrasted with previously reported results obtained for cylindrical samples where a scaling $<I_{t y p}>\sim w^{-2}$ has been found. Possible links to, up to date, unexplained experimental data are finally discussed.
\end{abstract}

Modern advances in nanotechnology made possible to manufacture systems in the submicron range. For those structures and at temperatures of few $m K$, the quantum mechanical coherence of the electronic wave functions dominates the transport of charge [1].

Recently, billiards with rough boundaries have been considered in order to analyze the effect of generic boundary deformations on the spectral and eigenfunction properties of ballistic samples [2 4 . In addition, during the last years, the magnetic response and the persistent currents of integrable and chaotic billiards in the presence of a magnetic flux have been the object of many research papers [5]6]. Most of them were inspired in the experiments of Ref. [7,8] in which the magnetic susceptibility and the persistent currents of mesoscopic semiconductor samples were measured. Continuous billiard systems posses an energy dispersion relation quadratic in the wave number $k$ and are reasonable models to describe semiconductor quantum dots in the ballistic regime. The situation is qualitative different for metallic systems. The breaking of the continuous translational symmetry by the lattice leads to a band structure and to a dispersion relation in $k$ which is equivalent to that of the free electron model only in the large wavelength limit. Moreover, the metallic regime is characterized by a half filled band and a typical electronic wavelength of the order of the lattice constant. Also and unlike high mobility heterostructures, a realistic model for a metallic sample should contemplate the effect of the elastic scattering with random impurities.

The observed persistent currents (PC) in metallic samples [9, 10] have been typically much larger than the predicted theoretical estimates that take into account the effect of static impurities and much effort is still devoted to understand the nature of the discrepancy between theory and experiments 11. In a recent work we have shown that by effect of confinement, large values of PC can arise in the half filling regime for clean metallic cavities of different shapes [12].

In this letter we address the effect of bulk disorder in confined metallic systems threaded by a magnetic flux. We are mainly concerned with the interplay between confinement and disorder effects and in particular with the sensitivity of the persistent current to the strength of disorder $w$. Our main result is that even deep in the diffusive regime large values of persistent currents can still be obtained for a wide variety of sample shapes.

We employ the spinless tight-binding hamiltonian defined on a square lattice and introduced in Ref. [12], with the additional inclusion of diagonal disorder. Once the profile of the sample is defined, the on-site energies are taken randomly distributed in the interval $[-w / 2, w / 2]$. We consider non-single connected loops of various shapes with up to 3000 sites and values of $w$ ranging from 0.02 to 5 in order to study the transition between the ballistic and the diffusive transport regimes. In the following all the lengths will be in units of the lattice parameter $a$, and the magnetic flux $\alpha$ will be measured in units of $\phi_{o}=h c / e$. An isolated non-single connected sample threaded by a magnetic flux $\alpha$ carries a persistent current $I$, even in the case of multiple scattering by static impurities. For a sample containing $N$ non-interacting electrons, the total current can be calculated at zero temperature as $I \sim-\partial E / \partial \alpha$ with $E$ the total energy of the system [13]. In the absence of disorder, the typical current $I_{t y p} \equiv \sqrt{\int_{0}^{1} I^{2} d \alpha}$ gives a measure of the magnitude of the current and is a (fluctuating) function of $N$. We denote its value at half filling $I_{o}$.

For a finite value of $w$, we define the average typical current $\left\langle I_{t y p}\right\rangle \equiv \sqrt{\left\langle I_{t y p}^{2}\right\rangle_{\delta n}}$ performing averages in a small energy window containing $1<<\delta n<<N$ levels. It was previously checked that this procedure is equivalent to average at the same time on the disorder configuration $(w)$ and on the number of levels $(\delta n)$ that is, $\langle\ldots\rangle_{w, \delta n}=\langle\ldots\rangle_{\delta n}$ [14].

Before considering disorder effects, we summarize the main results obtained for clean geometries. In Ref. 12] we show that for annular and square loops the corre- 
sponding density of states (DOS) has a local peak close to the band center $(E=0)$ at zero magnetic flux. In the absence of surface roughness (SR), the peak consists of border states that have in general large values of mean angular momentum. These states contribute to enhance in one or two orders of magnitude the value of $I_{o}$ with respect to values obtained for geometries with the same number of sites but without border states. The peak in the DOS is robust to changes in the size of the sample while preserving its shape. In addition, we here present numerical evidence supporting the conjecture that the peak in the DOS is present when the symmetry of the underlying lattice conforms a symmetry subgroup of the confining potential. This is illustrated in Fig. 1, where the spectra for four different samples are shown close to half filling: (a) square SL, (b) annular AL, (c) octagonal OL and (d) pentagonal PL loops 16]. In Figs. 1 (a), (b) and (c), a bunch of degenerate levels is observed at zero flux for $E=0$. These levels evolve paramagnetically for finite $\alpha$, generating large values of PC . Due to absence of crossings between occupied and empty levels close to half filling, $I_{t y p}$ increases monotonically with the number of particles [12]. The PL, Fig.1 (d), corresponds to a generic geometry where the mentioned symmetry condition is not fulfilled. There is no bunch of states at zero flux, and therefore no enhancement of the PC near half filling is expected.

When the confining potential has not the square or rectangular rotational symmetries our samples posses surface roughness (SR). This is the case for the AL and the OL in which, besides the border states that carry large currents, we find localized states in the bunch of degenerate levels at zero flux. These are states trapped by the SR and are not sensitive to the external magnetic flux. The flat lines on the top of the spectra of Figs. 1 (b) and (c) correspond to these states. A remarkable feature is that the charge distribution for the border states seems to be highly concentrated on classical periodic trajectories that bounce off the borders of the loops. We find, as in quantum billiard models, border states that look like resonant states associated to a continuous set of non isolated periodic orbits [17. In the left panel of Fig.2 (a) we show for the square loop SL, the contour plot of the absolute squared value of the wave functions $\left(|\Psi(x, y)|^{2}\right)$ for the $20^{\text {th }}$ state below half filling for $\alpha=0.025$. In the right panel of Fig. 2 (a) the family of classical orbits that supports the quantum state is drawn for comparison. Fig.2 (b) shows for the annular loop AL the contour plot for a quantum state with energy close to zero (that is belonging to the peak in the DOS) and with the charge distribution extended along a diamond-like orbit that goes around the sample with large mean angular momentum. As a consequence of the SR there is not a complete family of trajectories that supports the quantum state like in the square loop.

In summary we have found, as a consequence of the symmetry properties of the confining potential and the underlying lattice, clean metallic geometries with large values of $\mathrm{PC}$ at half filling. We name these kind of samples symmetric loops. However, a realistic model for a metallic cavity should contemplate the inclusion of bulk disorder in order to mimic the diffusive transport regime.

Sustitutional disorder introduces in the model a new energy scale that competes against the existence of border states carrying anomalously large currents. In order to study disorder effects on the PC it is necessary to determine the range of values of disorder $w$ that correspond to the diffusive regime in our samples. We begin analyzing the scaling of $\left\langle I_{t y p}>\right.$ with $w$ for the SL. According to elementary scattering theory a scaling as $<I_{\text {typ }}>\sim w^{-2}$ is predicted in the diffusive regime [14]. In Fig. 3(a) we show with square symbols the Log-Log plot of $\left\langle I_{t y p}>\right.$ at half filling as a function of $w$ for the SL in which the total number of sites is $N=2688$. We clearly observe a range of values of $w$ between 1 and 4, before the exponential damping characterizing the onset of the localized regime, in which the scaling is $<I_{\text {typ }}>\sim w^{-\gamma}$, with $\gamma=1.98$ obtained from the best fit. Therefore, this range of $w$ should correspond to the diffusive regime for other geometries containing the same number of total sites. Indeed we have obtained in the same range of values of $w$ a similar scaling law for a rectangular loop with $N=2424$ (both rectangles in the loop having the same aspect ratio), even though this loop is not a symmetric loop (in the sense defined above). Numerical calculations performed on cylindrical geometries satisfied the scaling law $<I_{\text {typ }}>\sim w^{-2} 114,15$.

On the other hand for samples with SR like the annular loop AL, the dependence of $\left\langle I_{t y p}\right\rangle$ at half filling with $w$ shows a non monotonic behavior that changes the overall value of the exponent to $\gamma \neq 2$ (see the circles in Fig. 3(a)). We argue that by effect of the bulk disorder (and before the onset of the localized regime) the states trapped by the SR are unpined being able to carry a finite PC. Moreover, there is no reason a priori to expect the same degree of response to a perturbation for all the trapped states. This explains the step-like behavior of $\left\langle I_{t y p}\right\rangle$ with $w$ observed in Fig. 3(a) for the AL. This non-monotonic response of the PC to disorder translates, in the case of the annular sample, to an average exponent $\gamma \approx 1.3$, significantly lower than in the case of the square loop SL. As a consequence, as can be observed from Fig. 3(a) there is range of values $w$ where the $\mathrm{PC}$ in diffusive annular loops are larger than those in square loops, despite the SR present in the former samples. This overall change in the exponent characterizing the diffusive regime does not seem to be a particular attribute of samples with SR. For other nonintegrable geometries without SR, like rectangular loops where the inner an outer border have different shapes (chaotic geometry), we also find an anomalous scaling $<I_{\text {typ }}>\sim w^{-\gamma}$ with $\gamma<2$. Nevertheless in the absence 
of SR we do not observe the non-monotonic response characteristic of samples with SR. Fig. 3(b) shows the Log-Log plot of $\left\langle I_{t y p}>\right.$ as a function of $w$ for a nonsymmetric rectangular loop formed by an inner rectangle of sides $l_{1}=13$ and $l_{2}=8$ and an external rectangle of sides $L_{1}=53$ and $L_{2}=48$, respectively. The obtained best fit is $<I_{\text {typ }}>\sim w^{-1.54}$. We stress that in the absence of disorder this geometry does not present border states because the symmetry condition that leads to the peak in the DOS is not satisfied. In other words, $I_{o}$ for a clean non-symmetric loop (see Fig. 1 dotted line) is much smaller than $I_{o}$ for clean symmetric loops (see inset of Fig. (4). Even more, $I_{t y p}$ for diffusive symmetric loops can be of the same order of magnitude than $I_{t y p}$ for a clean non-symmetric loop, as it is shown in Fig. 4 for a clean pentagonal loop PL and an annular loop AL with $w=1$.

In order to determine for a given diffusive sample the value of $\left\langle I_{t y p}>\right.$ at half filling, two factors must be considered: the value of the current in the clean system $\left(I_{o}\right)$ and the correction factor that takes into account the effect of disorder. For generic non-symmetric systems, the exponent $\gamma$ that takes into account the diffusive corrections is $\gamma<2$ but, as we previously explained, no enhancement of the value of $I_{o}$ is expected. On the other hand, in clean symmetric loops without SR due to the presence of border states, large values of $I_{O}$ can be obtained, although in the diffusive regime the exponent is $\gamma=2$. It is for clean symmetric loops with SR, that we find an optimal interplay between geometrical effects and bulk disorder. That is large values of $I_{o}$ compared to those of generic samples, and an anomalous scaling $<I_{\text {typ }}>\sim w^{-\gamma}$ with $\gamma<2$. Therefore these systems are candidates to have the largest values of persistent currents even deep in the diffusive regime. Also, we obtain that in symmetric loops (with or without SR) the response of the system to the external flux is always paramagnetic, even in the difussive regime.

The novel effects described in this work can be related to up to date unexplained experimental data on PC in mesoscopic metallic rings. In Ref. [9] rectangular and circular $A u$ loops with a large number of channels were designed and the PC measured. The corresponding signals were paramagnetic and more than an order of magnitude larger than predicted values that include the diffusive correction factor. In addition, in that experiment the reported value of the signal for the annular loops was 5 times larger than for the rectangular loop (although the estimated theoretical values were roughly the same). We believe that in order to model the samples employed in this experiment, geometries with decoupled degrees of freedom (like the cylinders employed in many theoretical papers) are not appropriate. We present numerical evidence showing that by breaking the translational symmetry in all directions, it is possible to create at finite magnetic flux border states near half filling. This states coherently contribute with large values of PC. As we have previously shown, the confinement in general gives rise to geometrical disorder (SR) that competes with the sustitutional disorder. This results in a non-monotonic behavior of $\left\langle I_{t y p}>\right.$ with $w$ and an overall exponent $\gamma<2$. Therefore, we can obtain for these systems larger values of PC than in previous models. Moreover, we find that the symmetric annular loops can have in the diffusive regime a $\mathrm{PC}$ as larger as in generic clean systems. In addition we show that in generic non-integrable geometries without SR the exponent $\gamma$ is also $<2$, although the response of $\left\langle I_{t y p}\right\rangle$ with $w$ is monotonic due to the absence of trapped states in the clean regime. This anomalous scaling of $\left\langle I_{\text {typ }}\right\rangle$ with $w$ could be a quantum signature of the classical non-integrability of the loops. The absence of symmetries in the clean system makes the effect of the sustitutional disorder not such strong as in the case of systems that in the clean regime preserve some rotational symmetry. Indeed, for clean integrable geometries, like the squares and symmetric rectangular loops presented in this work, and also in cylinders [14], the exponent is $\gamma \sim 2$. We believe that due to recent technological developments it should be feasible to design samples with specific symmetries in which our predictions could be confirmed.

This work was partially supported by UBACYT (TW35-TX61), CONICET and Fundación Antorchas.

[1] L.P. Kouwenhoven et.al., in Mesoscopic Electron Transport, edited by L.P. Kouwenhoven, L. Sohn and G. Schön, NATO Advanced Study Institute, Ser. E, Vol. 345 (Kluwer, Dordrecht, 1997).

[2] E. Cuevas, E. Louis, J. A. Verges, Phys. Rev. Lett. 77, 1970 (1996).

[3] K. M. Frahm and D. L. Shepelyansky, Phys. Rev. Lett. 74, 1440 (1997); Phys. Rev. Lett.79, 1883 (1997).

[4] Y. M. Blanter, A. D. Mirlin, B. A. Muzykantskii, Phys. Rev. Lett. 80, 4161 (1998).

[5] F. von Oppen and E. K. Riedel, Phys. Rev. B 48, 9170 (1993).

[6] K. Richter, D. Ullmo and R. Jalabert, Phys. Rep. 276, (1996).

[7] L.P. Lévy, D. H. Reich, L. Pfeiffer, and K. West, Physica B189, 204 (1993)

[8] D. Mailly, C. Chapelier and A. Benoit, Phys. Rev. Lett. 70, 2020 (1993).

[9] V. Chandrasekar et. al, Phys. Rev. Lett. 67, 3578 (1991).

[10] P. Mohanty, Ann. Phys. (Leipzig) 8, 549 (1999).

[11] For a review see T. Guhr, A. Müller-Groeling and H. A. Weidenmüller, Physics Reports 299, 189 (1998).

[12] V. M .Apel, G. Chiappe and M. J. Sánchez, Phys. Rev. Lett.85, 4152 (2000).

[13] H. F Cheung, Y. Gefen and E. K. Riedel, IBM J. Res. 
Develop.32, 359 (1988).

[14] G. Montambaux, H. Bouchiat, D. Sigeti and R. Friesner Phys. Rev. B 42, R7647, (1990).

[15] H. F Cheung, E. K. Riedel and Y. Gefen, Phys. Rev. Lett.62, 587 (1989).

[16] For example, a square loop is a square cavity of side $L_{1}$ with a square hole of side $L_{2}<L_{1}$ in its center.

[17] R. Narevich, R. E. Prange and Oleg Zaitsev, Phys. Rev. E62, 2046 (2000). (a)
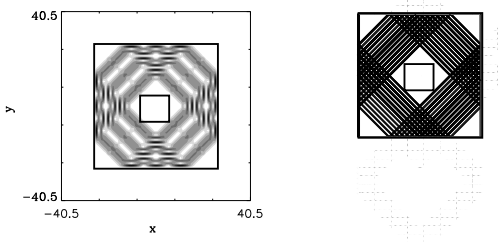

(b)
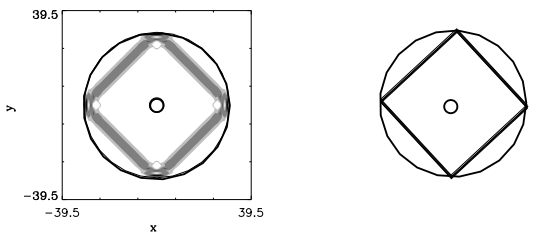

FIG. 2. (a) Contour plot of the absolute value square of the wave function for a border state of the SL for $\alpha=0.025$ (left panel). Sketch of the family of classical periodic orbits that support the quantum state (right panel). (b) diamond-like border state for the AL (left panel) togheter with the associated classical orbit (right panel). See the text for more details. (a)

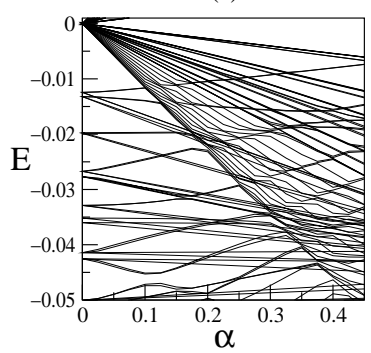

(c)

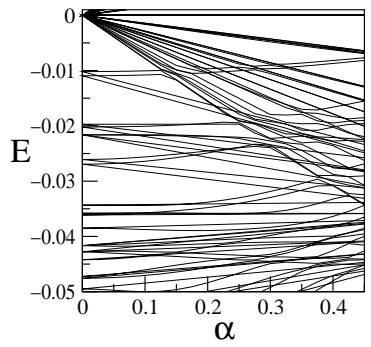

(b)
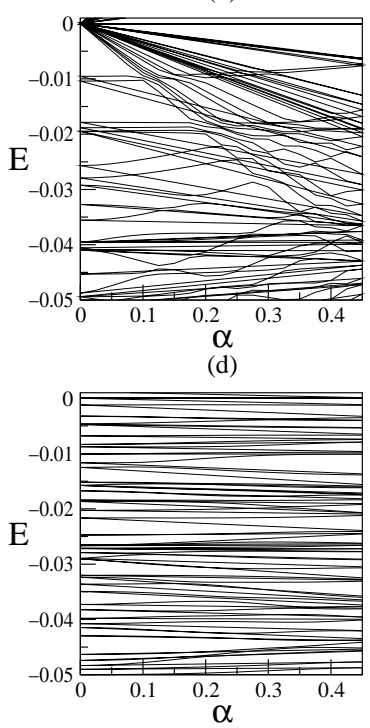

FIG. 1. Energy levels close to the band center as a function of $\alpha$ for: (a) SL in which the external (internal) square has a side $L_{1}=53\left(L_{2}=13\right)$, (b) an AL loop with external (internal) radius $R=30(r=3)$; (c) an OL loop with the inner (outer) octagon inscribed in a circumference of $r=3(R=32)$ and (d) a PL with the inner (outer) pentagon inscribed in a circumference of radius $r=3(R=35)$. 


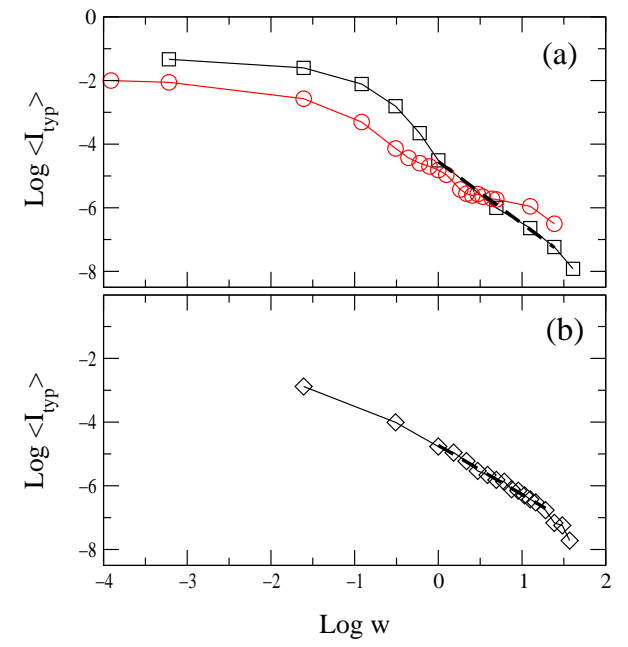

FIG. 3. (a): Log-Log plot of $\left\langle I_{t y p}\right\rangle$ as a function of $w$ for the AL (circles) and for the SL (squares). The dashed line shows the best fit $<I_{t y p}>\sim w^{-1.98}$ for the SL. (b): Idem but for a non-symmetric (chaotic) rectangular loop (diamonds) with the dashed line showing the best fit $\left\langle I_{\text {typ }}>\sim w^{-1.54}\right.$. See the text for details.

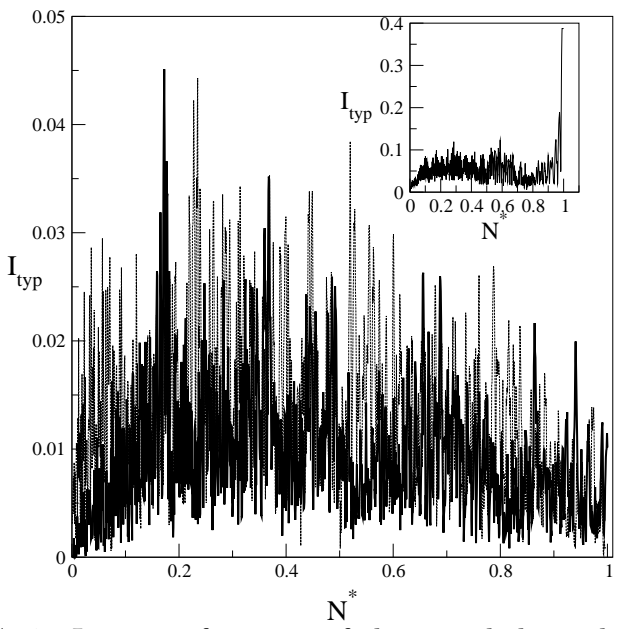

FIG. 4. $I_{\text {typ }}$ as a function of the rescaled number of levels $N^{*} \equiv n / N$ for the annular loop $\mathrm{AL}$ in the diffusive regime $w=1$ (thick solid line) and for the clean pentagon PL (thin dotted line). Both curves are almost superimposed. The inset shows for comparison $I_{t y p}$ as a function of $N^{*}$ for the clean $\mathrm{AL}$. 